\title{
A Case Report on the Challenging Diagnosis of Neuronal Ceroid Lipofuscinosis Type 2 (CLN2)
}

Journal of Inborn Errors of Metabolism \& Screening 2020, Volume 8: e20200010 DOI: 10.1590/2326-4594-JIEMS-2020-0010

\author{
Andrea Nunes', Joanna Meira ${ }^{2,3}$, Caio Cunha ${ }^{3}$, Marielza Veiga ${ }^{4}$, \\ Ana Paula Scholz de Magalhães ${ }^{5}$, Diana Rojas Málaga, \\ Roberto Giugliani ${ }^{5,6}$, Emília Katiane Embiruçu Leão ${ }^{2,3}$ ()
}

\begin{abstract}
Neuronal ceroid lipofuscinoses (NCLs), also referred as "Batten disease", are a group of thirteen rare genetic conditions, which are part of the lysosomal storage disorders. CLN type 2 (CLN2) is caused by the deficient activity of the tripeptidyl peptidase I (TPP1) enzyme, encoded by the TPP1 gene, most frequently leading to the classic late infantile phenotype. Nearly 140 CLN2causing mutations have been described. In this case report, we describe the identification of a new disease-causing mutation and highlight the importance of appropriate laboratory investigation based on clinical suspicion. The collection of dried blood spots (DBS) on filter paper, which is a convenient sample, can be used to measure the TPP1 enzyme activity and detect CLN2-related mutations. Since the biochemical and genetic diagnoses are possible and as the disease progression is fast and the therapeutic window is short, the investigation of CLN2 should be always considered when this diagnostic hypothesis is raised in order to enable the patients to benefit from the specific pharmacological treatment.
\end{abstract}

\section{Keywords:}

Lysosomal Storage Disorders, Neuronal Ceroid Lipofuscinoses, CLN2, TPP1, Childhood neurodegenerative diseases.

\section{Introduction}

Neuronal ceroid lipofuscinoses (NCLs), also referred as "Batten disease", are a group of thirteen neurodegenerative diseases associated with neuronal loss in the cerebellar and cerebral cortex with the accumulation of intralysosomal lipopigments. These genetic conditions are mainly inherited by an autosomal recessive manner [1-3].

CLN type 2 (CLN2) usually leads to a classic late infantile phenotype. The onset of clinical manifestations is between two and four years of age and includes psychomotor regression, speech delay, refractory epilepsy, myoclonus and ataxia. The diagnosis is confirmed by the finding of low activity of the tripeptidyl peptidase I enzyme (TPP1) in dried blood spot (DBS), leucocytes or cultured fibroblasts, and by the identification of a pathogenic mutation in each of the alleles of the TPP1 gene [4-6].

This case report details the diagnostic journey of a patient who presented with speech deterioration, refractory epilepsy and ataxia in the absence of photoparoxysmal response during electroencephalographic evaluations, in whom the diagnosis of late infantile CLN2 was eventually confirmed.

\footnotetext{
${ }^{1}$ Serviço de Neuropediatria, Hospital Martagão Gesteira, Salvador, BA, Brazil. ${ }^{2}$ Universidade Federal da Bahia, Serviço de Genética Médica, Complexo Hospital Professor Edgard Santos, Salvador, BA, Brazil.

${ }^{3}$ Universidade do Estado da Bahia, Departamento de Ciências da Vida, Salvador, BA, Brazil.

${ }^{4}$ Universidade Federal da Bahia, Serviço de Neurofisiologia, Complexo Hospital Professor Edgard Santos. Salvador, BA, Brazil.

${ }^{5}$ Hospital de Clínicas de Porto Alegre, Serviço de Genética Médica, Porto Alegre, RS, Brazil.

${ }^{6}$ Universidade Federal do Rio Grande do Sul, Departamento de Genética, Porto Alegre, RS, Brazil.
}

Received August 10, 2020. Revised form October 15, 2020. Accepted for publication October 22, 2020.

\section{Corresponding Author:}

Emília Katiane Embiruçu Leão. Universidade Federal da Bahia, Serviço de Genética Médica, Complexo Hospital Professor Edgard Santos, Salvador, BA, Brazil.

E-mail: ekeleao@yahoo.com.br 


\section{Case Report}

A seven-year-old male patient, born from a non-consanguineous couple, presented with unaltered development until three years of age, when he displayed refractory epilepsy characterized by generalized tonic-clonic, focal motor, and absence seizures, progressing to daily myoclonic-atonic seizures. Ataxia and neuropsychomotor regression of development were also observed from the age of 4.5 years, when treatment with valproic acid was introduced.

The patient underwent three electroencephalographic (EEG) that were performed using standardized methods with recording time greater than 40 minutes awake and sleep periods and intermittent photic stimulation was performed with low frequencies of 1-3 Hz. EEG evaluations as follows: a) at the age of 4 years, EEG showed absence of sleep spindles and spike-wave discharges in the posterior region of the brain; $b$ ) at the age of 6 years, the second EEG showed a worsening of the background activity of sleep and an increase in epileptiform discharges with a multifocal pattern, in addition to generalized spike-waves and c) at the age of 7 years, the third EEG showed a more pronounced

\section{A}

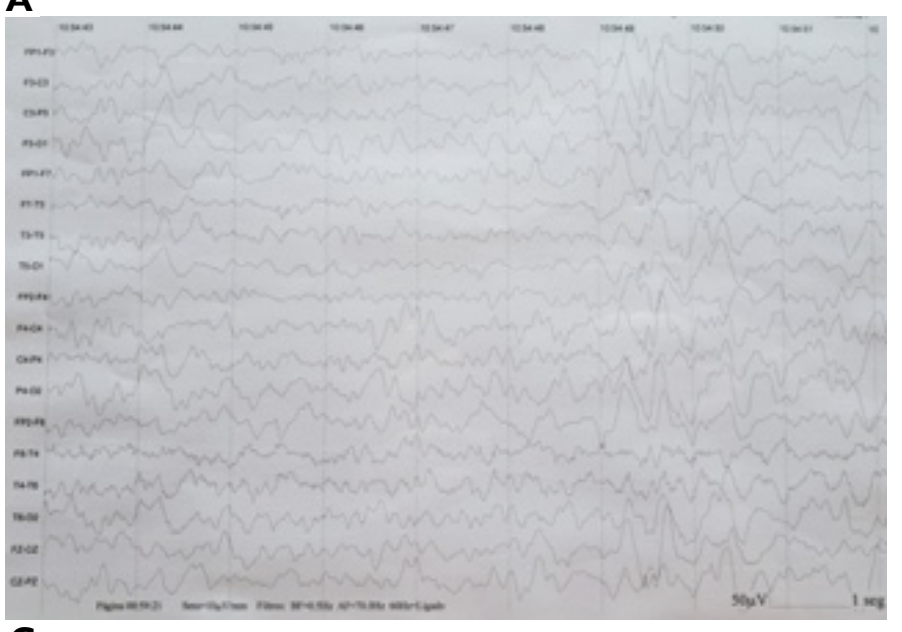

C

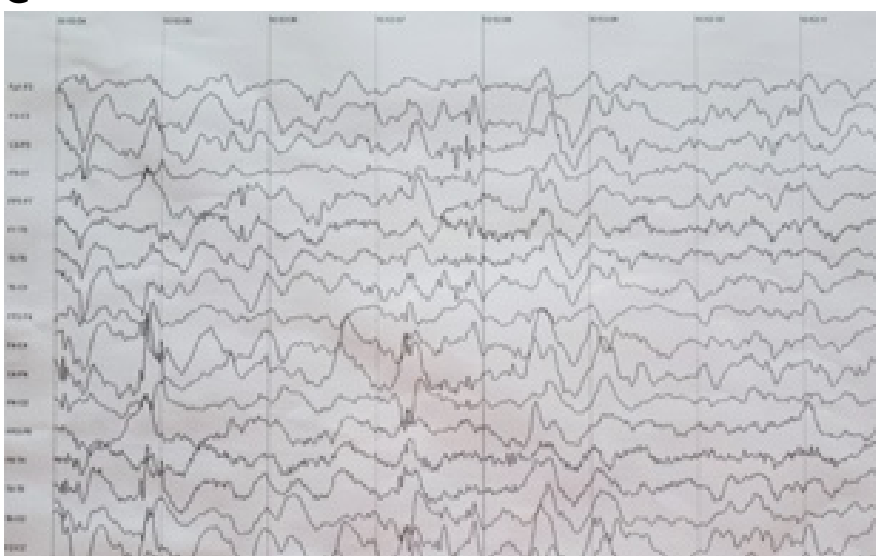

increase in generalized epileptiform discharges followed by a transient attenuation of background activity (Figure 1). Of note, photoparoxysmal response was absent in all the three EEGs.

Based on the clinical evaluation and on the tests performed, the suspicion of a CLN disease was raised and the next step was the measurement of the activities of palmitoyl protein thioesterase 1 (PTT1) and TPP1 enzymes in DBS, to screen for CLN1 and CLN2, respectively. These enzyme evaluations were performed when the patient was at 5 years and 6 months of age. The results revealed a reduced enzymatic activity of TTP1 (0.4 $\mathrm{nmol} / \mathrm{h} / \mathrm{ml}$; normal range from 4.0 to $23 \mathrm{nmol} / \mathrm{h} / \mathrm{ml}$ ) and mild reduction in PTT1 $(7.1 \mathrm{nmol} / \mathrm{h} / \mathrm{ml}$ of a normal range from 9.4 to $11 \mathrm{nmol} / \mathrm{h} / \mathrm{ml}$ ).

To confirm this finding, a targeted next-generation sequencing (NGS) approach was performed, using DNA isolated from DBS. A gene panel including several epilepsyrelated genes revealed the presence of c.225G $>$ A (p.Gln75Gln), a previously splice-altering CLN2-pathogenic variant in the exon 3, and c.503_504insTGGA (p.Phe169fs) in the exon 5 of TPP1 gene (NM_000391.4), both in heterozygous fashion and confirmed by Sanger sequencing. Unlike the first variant,

B

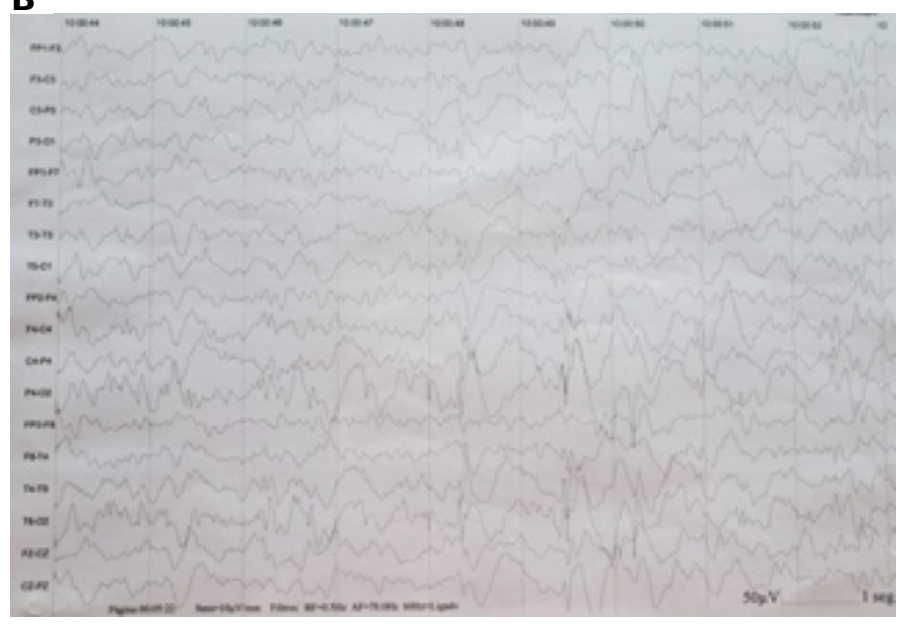

D

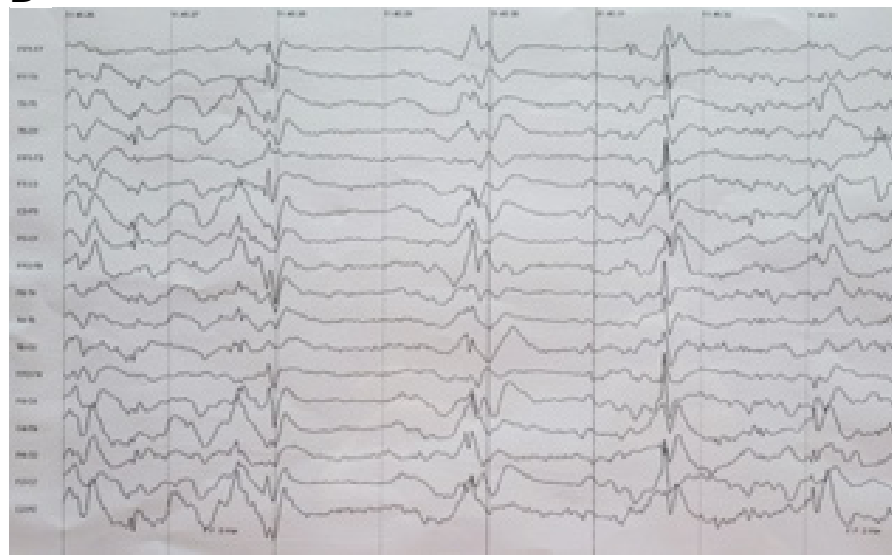

Figure 1. Electroencephalographic evaluation (EEG) in the CLN2 patient. All EEG showed slow background activity with absence of spindles during sleeping record and absence of alpha rhythm during awake record. At 1 year after disease onset, independent parietooccipital spikes on both sides in waking stage (Panel A), and during sleep (Panel B). At 3 years after disease onset, multifocal spikes were recorded (Panels C). At 4 years after disease onset, multifocal spikes and frequent generalized spike wave with attenuated segments (Panel D). 
the second correspond to a variant not previously described in the current online CLN-disease Database Mutation Table $(7,8)$. This new variant was classified as pathogenic following the American College of Medical Genetics (ACMG) criteria: PVS1 (very strong), PM2 (moderate) and PP3 (supporting) [9]. In silico analysis in Mutation Taster, SIFT Indel and VEP tool suggested this variant is pathogenic (10-12). Subsequently, the genetic analysis was also carried out in the patient's parents. The heterozygous variant c.225A $>\mathrm{G}$ was identified in the patient's father and the variant c.503_504insTGGA was identified in the patient's mother, evidencing that the mutations were inherited in trans in the index case.

An evaluation of TPP1 activity in leukocytes was also performed when the patient was 7 years-old $(5.3 \mathrm{nmol} / \mathrm{h} / \mathrm{mg}$, with normal range from 93 to $521 \mathrm{nmol} / \mathrm{h} / \mathrm{mg}$ in leukocytes), confirming the enzyme deficiency and corroborating the results previously obtained using DBS.

Although clinical worsening was progressive and significant, the most recent EEG with low frequency photostimulation showed no significant alterations in comparison with the previous evaluation.

At the most recent evaluation (at 8 years of age), the patient was restricted to bed without active interactions with the environment or other people. The patient displayed lack of speech, apathy, axial-predominant hypotonia, tetraparesis with hyperreflexia and bilateral signs of pyramidal release such as clonus and Babinski sign. Gastrostomy has been performed for nutritional support. Due to the progression of the disease, only supportive treatment is currently provided and includes levetiracetam, clobazam, lamotrigine and phenobarbital to reduce the frequency of epileptic seizures.

\section{Discussion}

The association of epileptic encephalopathy with predominance of myoclonic seizures and rapid and progressive neuropsychomotor regression from the 3 years of age should prompt the investigation of late infantile phenotype of NCLs, since they constitute the most common cause of childhood cognitive decline [13]. Due to the rapid neurodegenerative progression of NCLs, particularly CLN2, early detection is critical to ensure that patients may benefit from the specific treatment, which is currently available [14]. However, in spite of the limited awareness of CLN2, the clinical manifestations (speech delay, seizures and ataxia) are non-specific signs and may be found in other neurological disorders, thus contributing to misdiagnosis [15].

When facing clinical signs of NCLs, both PTT1 and TPP1 enzymatic activities in leukocytes, fibroblasts or DBS should be performed to investigate the clinical suspicion of CLN1 or CLN2, respectively. The reduction of TPP1 is the gold standard for laboratory diagnosis CLN2. In addition, the use of epilepsy gene panels for investigating unexplained seizures in childhood may also constitute a very helpful tool in the diagnosis. Finally, the detection of two pathogenic mutations in trans in the TPP1 gene can also be used for diagnosing CLN2 [4].

Herein, the samples for the evaluation of enzymatic activity and NGS were obtained by DBS, which is a convenient method to collect, store and ship blood samples for analysis in a reference laboratory. The possibility of running biochemical and genetic analysis using the same DBS sample allow the optimization of tests and may contribute to accelerate the diagnosis. In the light of the availability of cerliponase alfa, an accelerate diagnosis is critical for allowing patient benefit from this specific treatment [15].

As both enzymes evaluated were reduced (slight reduction of PPT1 and marked reduction of TPP1), genetic testing allowed the detection of two pathogenic mutations in the TPP1 gene, inherited from each of the parents. From the best of our knowledge, this is the first time that the mutation c.503_504insTGGA has been identified. Its pathogenicity is indicated by the decreased levels of TPP1 enzyme activity, identified in DBS and confirmed in leukocytes.

EEG is one of the most informative tests when CLN2 is suspected in children presenting with speech delay or neurological regression, in association with epilepsy. The most prominent abnormal finding in the EEG is the absence of sleep spindles, which reflects the onset of a disorganization of brain electrical activity [3]. In accordance, this was the first alteration found in the EEG of the patient. Additionally, the presence of photoparoxysmal response at low frequencies $(1-3 \mathrm{~Hz})$ is also an early (43\%) and frequent (73\% to $93 \%$ ) finding, though not display by all patients with CLN2. It should be noted that the standardization for the investigation of the photoparoxysmal response in photosensitive individuals advocates for starting the photostimulation with low frequencies (16-18). While the presence of photoparoxysmal response at low frequencies may constitute an indicative sign of CLN2, its absence does not discard the disease.

In this case, the patient did not display photoparoxysmal response in any of the performed evaluations, what may have contributed to delay the diagnosis. Importantly, the evolution of the patient's EEG tracing showed a progressive increase in the epileptogenic activity, initially with focal discharges, then multifocal, and finally with a predominance of generalized epileptiform discharges. Such progressive deterioration of the EEG activity was accompanied by a clinical worsening, compatible with the neurodegenerative progression of the disease.

Recent studies have highlighted an imagining pattern in the brain MRI of patients with CLN2. More specifically, thalamic hipointensity, thinning of the corpus callosum and hyperintensity in both the insular cortex and in the posterior limb of the internal capsule, in addition to the cerebellar and the brain cortical atrophy, may be verified in patients this disease. It should be mentioned however that these findings may be 
absent in patients in the initial stage of CLN2, as described by Aydin [19]. Cerebellar and brain cortical atrophies are not specific findings of NCLs as they are also found in other neurodegenerative diseases, such as progressive myoclonic epilepsies [20]. More evidence investigating the association of CLN2 progression and the imaging findings may contribute the elucidate the role of imaging exams in the diagnosis and follow-up in CLN2 [18, 19, 21]. In the patient of the present case, MRI was unaltered in the first months after the disease onset. In the posterior analyses, cerebral and brain cortical atrophy were identified.

There is a specific pharmacological treatment approved for CLN2: the enzyme replacement therapy with human tripeptidyl peptidase 1 (cerliponase alfa), administered by intraventricular route every two weeks. However, there is a lack of evidence supporting the treatment when the disease is already advanced. This can be evaluated by the CLN2 Clinical Rating Scale, a score on the motor and language domains ranging from 0 to 6 , with 0 representing no function and 3 representing normal function in each of the two domains [22]. In the present case, the interval between the symptom's onset (3-years-old) and the diagnosis (7-years-old) was four years, making the enzyme replacement therapy probably not effective, since the patient obtained 0 in the CLN2 Clinical Assessment Scale.

In the pediatrics age, NCLs are considered the most prevalent neurodegenerative diseases in children [23]. The evaluation of the enzymatic activity of PTT1 (CLN1), TPP1 (CLN2), and the presence of occipital paroxysms on EEG performed with low frequency photostimulation can corroborate the suspicion [4]. Although enzyme assays are useful to screen for CLN1 and CLN2, genetic analysis evaluation through gene panels or whole exome sequencing is needed for diagnosis the majority of NCLs since they are not associated with an enzyme deficiency and the phenotypes of CLN2 may be similar [23].

As evidenced by the case, the interval between the onset of symptoms and diagnosis should be as short as possible, due to the rapidly progressive neurodegenerative nature of the disease [6]. In fact, the specific pharmacological treatment can be initiated in patients who achieve three points in the Hamburg scale, which assesses the functional ability of the patients [22]. In this regard, DBS constitute an interesting tool, as it is convenient to collect, store and ship and allows the evaluation of both enzymatic activity and genetic pattern in the same sample. Despite the assay, TPP1 enzyme activity in DBS should always be considered as a screening test since it is a simple procedure with convenient collection, handling and transportation that should be considered anytime that CLN2 is suspected. The diagnosis should be confirmed by the observation of a reduced TPP1 activity in leukocytes and/or by the finding of a pathogenic mutation in each of the alleles of the TPP1 gene.

The present case report reinforces the importance of the medical journey of the patient and the knowledge of the natural history of CLNs. In the light of an unspecific EEG and brain MRI, the refractory seizures associated with neurodevelopmental regression (predominately cognitive decline and ataxia) raised the hypothesis of CLN2, a catastrophic disease with a specific treatment currently available.

\section{Acknowledgments}

The authors would like to thank Elisa Sobreira, MD and Debora Mesojedovas, PharmD for contributions. A special thanks to Stream Medical Affairs and Camilla Patti Hissamura for providing medical writing assistance to the authors in the preparation of this manuscript.

\section{Funding}

BioMarin Brasil Farmacêutica LTDA sponsored the medical writing support for this case report. Authors are entirely responsible for the scientific content of this manuscript.

\section{Declaration of Conflicting Interests}

AN, JM, CC, MV, APSM, DRM, RG and EKEL declare no conflict of interest.

\section{Ethics Approval and Consent to Participate}

The Ethical Committee from the Hospital Universitário Professor Edgard Santos/UFBA approved the study under the CAAE \#49581915.0.0000.0049 number protocol.

\section{Authors' Contributions}

AN, JM, CC, MV, APSM, DRM, RG and EKEL conceived the manuscript and drafted the Portuguese version. RG and EKEL revised the text critically. All authors read and approved the final manuscript.

\section{References}

1. Haltia M, Goebel HH. The neuronal ceroid-lipofuscinoses: a historical introduction. Biochim Biophys Acta. 2013;1832(11):1795-1800. doi: 10.1016/j.bbadis.2012.08.012

2. Mink JW, Augustine EF, Adams HR, Marshall FJ, Kwon JM. Classification and natural history of the neuronal ceroid lipofuscinoses. J Child Neurol. 2013;28(9):1101-1105. doi: 10.1177/0883073813494268

3. Nita DA, Mole SE, Minassian BA. Neuronal ceroid lipofuscinoses. Epileptic Disord. 2016;18(S2):73-88. doi: 10.1684/epd.2016.0844

4. Fietz M, AlSayed M, Burke D, Cohen-Pfeffer J, Cooper JD, Dvořáková $\mathrm{L}$, et al. Diagnosis of neuronal ceroid lipofuscinosis type 2 (CLN2 disease): Expert recommendations for early detection and laboratory diagnosis. Mol Genet Metab. 2016;119(1-2):160-167. doi: 10.1016/j.ymgme.2016.07.011 
5. Williams RE, Adams HR, Blohm M, Cohen-Pfeffer JL, de Los Reyes E, Denecke J, et al. Management Strategies for CLN2 Disease. Pediatr Neurol. 2017;69:102-112. doi: 10.1016/j.pediatrneurol.2017.01.034

6. Nickel M, Simonati A, Jacoby D, Lezius S, Kilian D, Van de Graaf B, et al. Disease characteristics and progression in patients with late-infantile neuronal ceroid lipofuscinosis type 2 (CLN2) disease: an observational cohort study. Lancet Child Adolesc Health. 2018;2(8):582-590. doi: 10.1016/S2352-4642(18)30179-2

7. CLN2/TPP1. NCL Resource. Updated October 2018. Available from: https://www.ucl.ac.uk/ncl/ CLN2mutationtable.htm.

8. Gardner E, Bailey M, Schulz A, Aristorena M, Miller N, Mole SE. Mutation update: Review of TPP1 gene variants associated with neuronal ceroid lipofuscinosis CLN2 disease. Hum Mutat. 2019;40(11):1924-1938. doi: 10.1002/ humu. 23860

9. Richards S, Aziz N, Bale S, Bick D, Das S, Gastier-Foster $\mathrm{J}$, et al. Standards and guidelines for the interpretation of sequence variants: a joint consensus recommendation of the American College of Medical Genetics and Genomics and the Association for Molecular Pathology. Genet Med. 2015;17(5):405-424. doi: 10.1038/gim.2015.30

10. $\mathrm{Hu}$ J, Ng PC. SIFT Indel: predictions for the functional effects of amino acid insertions/deletions in proteins. PLoS One. 2013;8(10):e77940. doi: 10.1371/journal.pone.0077940

11. McLaren W, Gil L, Hunt SE, Riat HS, Ritchie GR, Thormann A, et al. The Ensembl Variant Effect Predictor. Genome Biol. 2016;17(1):122. doi: 10.1186/s13059-016-0974-4

12. Schwarz JM, Cooper DN, Schuelke M, Seelow D. MutationTaster2: mutation prediction for the deepsequencing age. Nat Methods. 2014;11(4):361-62. doi: 10.1038/nmeth.2890

13. Schulz A, Kohlschütter A, Mink J, Simonati A, Williams R. NCL diseases - clinical perspectives. Biochim Biophys Acta. 2013;1832(11):1801-1806. doi: 10.1016/j.bbadis.2013.04.008

14. Trivisano M, Specchio N. Red flags for neuronal ceroid lipofuscinosis type 2 disease. Dev Med Child Neurol. 2020;62(4):414. doi: 10.1111/dmcn.14389
15. Specchio N, Pietrafusa N, Trivisano M. Changing Times for CLN2 Disease: The Era of Enzyme Replacement Therapy. Ther Clin Risk Manag. 2020;16:213-222. doi: 10.2147/ TCRM.S241048

16. Specchio N, Bellusci M, Pietrafusa N, Trivisano M, de Palma L, Vigevano F. Photosensitivity is an early marker of neuronal ceroid lipofuscinosis type 2 disease. Epilepsia. 2017;58(8):1380-1388. doi: 10.1111/epi.13820

17. Kohan R, Carabelos MN, Xin W, Sims K, Guelbert N, Cismondi IA, et al. Neuronal ceroid lipofuscinosis type CLN2: a new rationale for the construction of phenotypic subgroups based on a survey of 25 cases in South America. Gene. 2013;516(1):114-121. doi: 10.1016/j.gene.2012.12.058

18. Johnson AM, Mandelstam S, Andrews I, Boysen K, YaplitoLee J, Fietz M, et al. Neuronal ceroid lipofuscinosis type 2: an Australian case series. J Paediatr Child Health. 2020;56(8):1210-1218. doi: 10.1111/jpc.14890

19. Aydın K, Havali C, Kartal A, Serdaroğlu A, Haspolat Ş. MRI in CLN2 disease patients: Subtle features that support an early diagnosis. Eur J Paediatr Neurol. 2020; 28:228-236. doi: 10.1016/j.ejpn.2020.07.009

20. Orsini A, Valetto A, Bertini V, Esposito M, Carli N, Minassian BA, et al. The best evidence for progressive myoclonic epilepsy: A pathway to precision therapy. Seizure. 2019;71:247-257. doi: 10.1016/j.seizure.2019.08.012

21. Biswas A, Krishnan P, Amirabadi A, Blaser S, MercimekAndrews S, Shroff M. Expanding the Neuroimaging Phenotype of Neuronal Ceroid Lipofuscinoses. Am J Neuroradiol. 2020;28:228-236. doi: 10.1016/j. ejpn.2020.07.009

22. Schulz A, Ajayi T, Specchio N, de Los Reyes E, Gissen P, Ballon D, et al. Study of Intraventricular Cerliponase Alfa for CLN2 Disease. N Engl J Med. 2018;378(20):1898-1907. doi: 10.1056/nejmoa1712649

23. Mukherjee AB, Appu AP, Sadhukhan T, Casey S, Mondal A, Zhang Z, et al. Emerging new roles of the lysosome and neuronal ceroid lipofuscinoses. Mol Neurodegener. 2019;14(1):4. doi: 10.1186/s13024-018-0300-6 